\title{
Segmentation of brain structures by watershed transform on tensorial morphological gradient of diffusion tensor imaging
}

\author{
Leticia Rittner*, Simone Appenzeller ${ }^{\dagger}$ and Roberto Lotufo* \\ ${ }^{*}$ School of Electrical and Computer Engineering \\ University of Campinas - UNICAMP, C.P. 6101, 13083-852, Campinas (SP), Brazil \\ lrittner@dca.fee.unicamp.br,www.dca.fee.unicamp.br/lotufo \\ ${ }^{\dagger}$ Department of Medicine, Rheumatology Unit \\ University of Campinas - UNICAMP, 13083-970, Campinas (SP), Brazil \\ appenzellersimone@yahoo.com
}

\begin{abstract}
Watershed transform on tensorial morphological gradient (TMG) is a new approach to segment diffusion tensor images (DTI). Since the TMG is able to express the tensorial dissimilarities in a single scalar image, the segmentation problem of DTI is then reduced to a scalar image segmentation problem. Therefore, it can be addressed by well-known segmentation techniques, such as the watershed transform. In other words, by computing the TMG of a DTI, and then using the hierarchical watershed transform, it is possible to segment brain structures, such as the corpus callosum, the ventricles and the cortico-spinal tracts, and use the results for subsequent quantitative analysis of DTI parameters. Experiments showed that segmentations obtained with the proposed approach are similar to the ones obtained by other segmentation techniques based on DTI and also segmentation methods based on other Magnetic Resonance Imaging (MRI) modalities. Since the proposed method, as opposed to the majority of the DTI based segmentation methods, does not require manual seed and/or surface placement, its results are highly repeatable. And unlike other methods that have sometimes four parameters to be adjusted, the only adjustable parameter is the number of regions in which the image should be segmented, making it simple and robust.
\end{abstract}

Keywords-Diffusion tensor imaging; MRI; Segmentation; Watershed transform; Mathematical morphology;

\section{INTRODUCTION}

Diffusion tensor imaging (DTI) is a relatively new Magnetic Resonance Imaging (MRI) modality of able to quantify the anisotropic diffusion of water molecules in highly structured biological tissues. It is unique in its non-invasive ability to quantify changes in neural tissue microstructure within the human brain.

Delineation of a region of interest (ROI) is usually necessary for performing subsequent quantitative analysis of DTI. However methods for delineate the ROI are normally manual and subjective, very time consuming and requiring considerable expertise.

Usually, when acquiring DT-MRI of a subject, other MR images are also acquired, such as $T 1$-weighted and $T 2$ weighted MRI. Therefore, one option to delineate a region of interest in DT images, is to perform the segmentation of structural images, such as $T 1$-weighted or $T 2$-weighted segmentation, for which several algorithms are available [1], [2], [3], [4], [5], [6], [7]. Then, by registering the DT image to the $T 1$ or $T 2$ image, is possible to use the result of the segmentation made on $T 1$ or $T 2$ to segment the DT image.

Without a doubt, it would be desirable to have a method that segment directly the DTI, instead of segmenting it indirectly, based on $T 1$ or $T 2$ weighted images. First of all, because it would not be necessary to acquire $T 1$ or $T 2$ weighted images. Second, because segmentation methods based on $T 1$ or $T 2$ are not trivial and are usually time consuming. In addition, registration of segmentation result to DT images introduces errors.

Initially several DTI segmentation methods were proposed to address the problem of finding individual fiber paths. Only in the last decade recent papers have addressed the problem of region based segmentation of DT-MRI.

Zhukov et al. in [8] applied a level-set modeling and segmentation techniques to the derived scalar volumes to create geometric models of specific brain structures. The geometric models were then used for quantitative analysis, including volume and surface area calculations. Their level set segmentation approach consisted of defining a set of suitable preprocessing techniques for initialization and selecting/tuning different feature-extracting terms in the level set equation to produce a surface deformation. Because level set models move using gradient descent, they seek local solutions, and therefore the results are strongly dependent on the initialization, i.e., the starting position of the surface. Additionally this method fails to distinguish between regions with same diffusion anisotropy magnitude but oriented in different directions.

Zhizhou and Vemuri [9] proposed also a DTI segmentation based on a level-set curve evolution technique, that operates on the diffusion tensor fields. However, these variational formulations with iterative gradient descent based solutions are sensitive to parameter settings and initialization, and hence may get stuck at suboptimal local minima of the energy functional. 
Another level-set based front-propagation scheme was presented by Jonasson et al. [10], in which the front propagates to a new voxel based on the similarity between the tensor at that voxel and a few tensors inside the current segment lying along the surface normal. Such an approach might encounter problems at sharp bends in thin tracts like, for example, the cingulum.

In [11], Weldeselassie and Hamarneh extended the graph cuts scalar field segmentation technique proposed by Boykov and Jolly [12] to DTI. In their extension, the graph vertices correspond to the tensor voxels in the DT-MR image and the graph connectivity edge weights are computed using tensor dissimilarity metrics. Seed points provided by the user give clues about the location of the object of interest and the background and often, the user needs to interactively modify the seed points in order to improve the segmentation results.

Lenglet et al. [13] presented a statistical surface evolution framework for the segmentation of diffusion tensor images and introduced a Riemannian distance measure between tensors, modeling each class by a single Gaussian in the Riemannian space. The proposed method extends their modeling approach by incorporating a generic nonparametric model for each class that is able to accurately model tensor statistics in fiber bundles.

Recently, Niogi et al. [14] proposed a semiautomated region-growing and chain-linking to produce 3D regions of interest of white matter tracts without explicitly performing fiber tracking. It fails to segment a structure that contains a lesion, since it will not select cerebrospinal fluid or gray matter as boundary pixels. And because it was designed to terminate segmentation when the principal direction of diffusion changes to an orthogonal direction, in structures with high curvature areas, its segmentation will terminate prematurely.

All cited algorithms require previous knowledge of the structure to be segmented and depend on seed placement, initial surface delineation and/or parameter adjustments, making the segmentation process very susceptible to initialization.

In order to reduce the influence of user choices, what this work proposes is a segmentation algorithm based on the watershed transform and the tensorial morphological gradient (TMG) of a diffusion tensor image (DTI). The tensorial morphological gradient was first applied to segment tensorial images representing color images [15] and then, its concept was extended and applied to synthetic diffusion tensor images [16]. It computes the tensorial morphological gradient based on the similarity between tensors and segments it using the watershed transform. The only parameter to be chosen is the number of regions in which the image should be segmented.

This paper is organized as follows: Section II describes the DTI segmentation algorithm based on TMG and compares it to other DTI segmentation methods . Section III presents the segmentation of brain structures, such as the corpus callosum, the ventricules and the cortico-spinal tract. Finally, conclusions are summarized in Section IV.

\section{DTI SEGMENTATION OF BRAIN STRUCTURES}

Diffusion tensor image segmentation is a challenging task exactly because it has as input a tensorial image and the segmentation method has to decide which information should be considered for segmentation purposes. Furthermore, either existing segmentation methods have to be adapted to deal with tensorial information or completely new segmentation methods have to be developed to accomplish this task. Alternatively, what this work proposes is the computation of a gradient, that transforms the diffusion tensor image into an scalar image, and makes possible the use of a wide range of existing segmentation methods in order to perform the segmentation. In this case, the segmentation method chosen to accomplish the task was the hierarchical watershed transform.

\section{A. TMG based segmentation}

The present work proposes the computation of the tensorial morphological gradient (TMG) of a diffusion tensor image (DTI) as the initial step to segment it. Inspired on the morphological gradient, a tensorial morphological gradient (TMG) was defined and first described in [15]. It was used at that time, together with a tensorial representation of colors, to segment color images. In [16], the concept was expanded, applying it to segment synthetic diffusion tensor images.

The morphological gradient is one of the several existing gradients used in image processing to detect edges and depends on the size and shape of the chosen structuring element. Using a flat structuring element at each point, the morphological gradient yields the difference between the maximum and the minimum values over the neighborhood at the point determined by the flat structuring element [17]. The use of a three-dimensional structuring element leads to a morphological gradient that takes into account the neighborhood in all directions, and not only in an specific plane.

Let $E=\mathbb{Z} \times \mathbb{Z}$ be the set of all points in the tensorial image $f$. The tensorial morphological gradient (TMG) is defined by

$$
\nabla_{B}^{T}(f)(x)=\bigvee_{y, z \in B_{x}} d_{n}\left(\mathbf{T}_{y}, \mathbf{T}_{z}\right)
$$

$\forall x \in E$, where $\bigvee$ is the supremum of a subset, $B \subset$ $E$ is a structured element centered at the origin of $E, d_{n}$ represents any of the similarity measures presented in DTI literature [18], [19], [20], [21], [9], [22], $\mathbf{T}_{y}$ is the tensor that represents the diffusion in $y$, and $\mathbf{T}_{z}$ is the tensor that represents the diffusion in $z$ ( $y$ and $z$ are in the neighborhood of $x$, defined by $E$ ). $\nabla_{B}^{T}$ is the proposed TMG. 
Once the tensorial information is translated into a scalar image, namely the TMG, the next step is to segment it using the watershed transform from markers [23], [24].

The critical part in the watershed segmentation is the proper choice of markers. The classical watershed places the markers automatically in each regional minima of the image. This usually leads to a over-segmentation and is not adequate for medical imaging applications. To solve the over-segmentation problem, hierarchical approaches are considered in order to retain the most significant regions of the image at different scales [25].

The dynamics of the regional minima have been used to build the hierarchy. Essentially, this hierarchy can be interpreted as a set of region adjacencies, wherein an ordering is determined by a valuation, which can be controlled by the relative altitudes of the regional minima. The contrast dynamics of a regional minimum is defined as the minimal climb required for a path starting from a regional minimum to reach another one with strictly lower altitude, the climb being the difference in altitude between the highest point of the path and the regional minimum under study. Regional minima with highest dynamics will be used as markers for watershed segmentation.

In this work, after calculating the TMG of the original image, the $n$ structures in the image which has the greatest volume extinction values are automatically selected. The $n$ markers assigned to these regions are then used in the watershed transform, which segmented the TMG in $n$ regions.

\section{B. Comparison to other DTI segmentation methods}

As described in Section I, a few region based segmentation of DT-MRI have been proposed in the last decade, most of them based on level-set, statistical surface evolution and region growing techniques. Some of them were designed to segment only fiber bundles, while others focused on segmenting white matter structures, such as the corpus callosum and the cortico-spinal tract.

Considering some of the most recent techniques that were proposed in the literature to segment the corpus callosum, an analysis of the requirements of each algorithm was done and summarized below, in an effort to demonstrate the strength of the proposed methodology regarding initialization and parameters dependency.

Jonasson et al. [10] proposed a front propagation algorithm to segment white matter fiber tract based on DTI. To perform the segmentation a initial surface has to be placed inside the tract to be segmented. The surface is then propagated using a chosen similarity measure. A curvature dependent speed is added to regularize the flow and its weighting parameter have to be assigned. Also, a threshold value for the speed is necessary, to prevent unwanted propagation.

In [13], Lenglet et al. proposed a surface evolution algorithm for DTI segmentation that also requires a initialization of the surface evolution. It addresses the segmentation problem as the maximization of a posteriori segmentation probability. There are two parameters that have to be chosen: the first one is the value of $\nu$ in the term that expresses the probability of the interface to represent the structure of interest. It constrains the smoothness of the surface and is usually set in the range 1 to 10 . The second parameter is a threshold for the variance which, whenever reached, induces the end of the update for the statistical parameters.

To segment a white matter structure by the semiautomated segmentation method proposed in [14], first one has to select a pixel within the structure to be segmented, that will be used as a sample of the structure. Second, the computation of a magnification array requires values selection for the three constants $\alpha, \beta$ and $\gamma$. Then, pixels are labeled, after being transformed by this magnification matrix and according to a threshold value, that also has to be chosen.

Table I summarizes the requirements and limitations of each segmentation method. While all the other DTI segmentation methods cited here require seed placement or initial surface delineation, there is no initialization needed in the proposed method. Moreover, the segmentation technique based on the TMG has as only input parameter the number of regions to segment, in opposition to 2 required parameters in [10] and [13], and 4 parameters in [14].

It is important to point out that the choice of a different number of regions $n$ does not mandatorily deteriorate the segmentation result. At best, the segmentation of the desired structure could be not affected at all. Otherwise, a lower number of regions would make the desired structure to be merged with an adjacent structure. In that case, the user would have to run the watershed again, increasing the number of regions. In the other hand, a higher number of regions would only cause the desired structure to receive two or more distinct labels instead of only one. In this case, the user would only have to group the respective labels in order to obtain the whole structure.

Table I

REQUIREMENTS OF DTI SEGMENTATION METHODS

\begin{tabular}{|lcccc|}
\hline & Jonasson & Lenglet & Niogi & $T M G$ \\
& {$[10]$} & {$[13]$} & {$[14]$} & \\
\hline Initial surface & yes & yes & no & no \\
Seed pixel & no & no & yes & no \\
Number of parameters & $2^{a}$ & $2^{b}$ & $4^{c}$ & $1^{d}$ \\
Postprocessing & no & no & yes & yes \\
Designed for & $\mathrm{WM}$ & $\mathrm{WM}$ & $\mathrm{WM}$ & all \\
\hline
\end{tabular}

${ }^{a}$ regularization weighting parameter and threshold for the speed

${ }^{b}$ smoothness of the surface and threshold for the variance

${ }^{c}$ constants of magnification array and threshold for diffusion components

${ }^{d}$ number of regions 


\section{RESULTS}

\section{A. MRI data acquisition and preprocessing}

The data used in our experiments were acquired on a Siemens 3T Trio MR scanner (Siemens Medical Systems, Erlangen, Germany) using an 8-channel phased array head coil. Diffusion encoding was achieved using a singleshot spin-echo echo planar sequence with twice-refocused balanced diffusion encoding gradients. The datasets acquired contained: a $T 1$ weighted image, 176 slices, $1 \mathrm{~mm}$ isotropic voxel size, $T E=2.98 \mathrm{~ms}, T R=2.3 \mathrm{~s}$; a $T 2$ weighted image, 77 slices, $1.0 \mathrm{~mm} \times 1.0 \mathrm{~mm} \times 2.0 \mathrm{~mm}$, $T E=84 \mathrm{~ms}, T R=14.6 \mathrm{~s}$ and a $P D$ image ${ }^{1}, 77$ slices, $1.0 \mathrm{~mm} \times 1.0 \mathrm{~mm} \times 2.0 \mathrm{~mm}, T E=19 \mathrm{~ms}, T R=3.2 \mathrm{~s} ;$ diffusion images with $N=30$ diffusion encoding directions with 63 slices, $2.0 \mathrm{~mm} \times 2.0 \mathrm{~mm} \times 2.0 \mathrm{~mm}, T E=95 \mathrm{~ms}$, $T R=8.7 \mathrm{~s}$ and $b=1000 \mathrm{~s} / \mathrm{mm}^{2}$ (the $\mathrm{b}$ value describes the degree of diffusion weighting, determined by the duration and strength of the sensitizing pulsed gradients in the MR experiment).

The diffusion data was first linearly interpolated before tensor estimation. The MINC tools from the Montreal Neurological Institute, McGill University, were used for all diffusion image preprocessing.

\section{B. TMG computation and segmentation results}

In our segmentation experiments the TMG of the DTI was computed using the Frobenius Norm as the similarity measure and the hierarchical watershed was then used to segment desired structures of the brain. The watershed transform, the extinction functions and other morphological functions used can be found in the "SDC Morphology Toolbox for MATLAB" [26]. The TMG computation and the segmentation algorithm was implemented in MATLAB 7.0 .

Fig. 1 shows one slice of the tensorial information, already cropped around the desired structure (corpus callosum) and overlayed on a $T 1$ weighted image. The information contained in each pixel in the front image is the ellipsoid representing each diffusion tensor, colored according to the orientation of the principal eigenvectors. Although it is possible to distinguish the corpus callosum by observing the ellipsoids in Fig. 1, it is not obvious to automatically segment it without giving any prior information, specially because one have to deal with a tensorial information, containing both magnitude and direction of the diffusion.

To automatically segment the corpus callosum shown in Fig. 1, first the tensorial morphological gradient (TMG) was computed using the Frobenius Norm as the tensorial similarity measure. The structuring element chosen to define the neighborhood to be considered in the TMG computation was

\footnotetext{
${ }^{1}$ An MR image dependent primarily on the density of protons in the imaging volume. Proton density contrast is a quantitative summary of the number of protons per unit tissue.
}

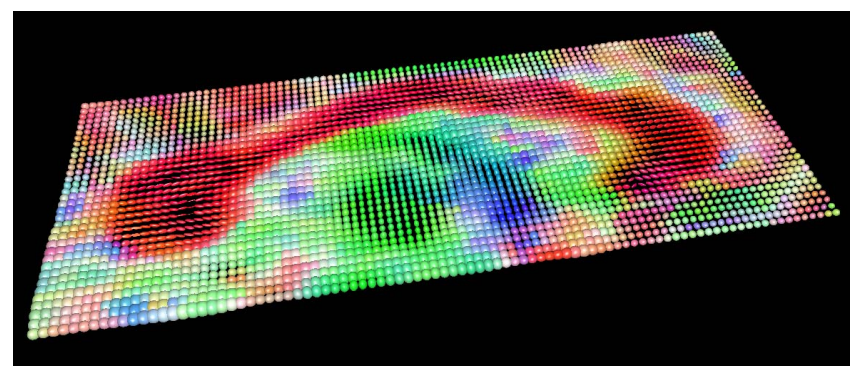

Figure 1. Ellipsoids representing the diffusion tensors in the region containing the corpus callosum

a 6-connected structuring element (3D). Once computed, the TMG was then used by the watershed transform to segment the corpus callosum. The used criteria to chose the watershed markers was to fix the number of regions, in which the image should be segmented. In this specific case, markers were imposed to the basins with the 60 greatest volume extinction values, i.e., the watershed operator segmented the TMG of the DTI in the 60 most significant regions.

Fig. 2 presents three steps of the segmentation process: Fig. 2(a) shows one slice of the computed TMG, Fig. 2(b) contains the same slice labeled according to the watershed result and Fig. 2(b) depicts finally the $3 D$ segmentation result. As can be seen in Fig. 2(a) the TMG was able to detect the borders of the corpus callosum, and the dark area inside it shows that the diffusion tensors inside this structure are very homogenous, leading to a very low gradient. Fig. 2(b) shows the labels assigned to one slice by the watershed transform, where each color represents one label. The watershed transform was able to assign one single label to the corpus callosum, in this case, the dark red area in the upper part of the image.

It is important to notice that, although Fig. 2(a) and Fig. 2(b) depicts only one slice of the TMG and of the watershed result, the TMG and the watershed transform were computed taking into account the three-dimensional information. This is guaranteed by the choice of the structuring element (in this case, a 6-connected structuring element). For another specific application or structure, a bi-dimensional structuring element could be more adequate. This could be easily chosen by the user without any modification of the algorithm. Finally, Fig. 2(c) shows the whole segmented volume (3D result), from which is possible to conclude that the hierarchical watershed successfully segmented the corpus callosum.

To confirm our segmentation result, we segmented the corpus callosum of the same subject using the ANIMAL+INSECT [7], an algorithm for improved automatic segmentation of gross anatomical structures of the human brain. It merges the output of a tissue classification process (INSECT) with gross anatomical region masks, automatically defined by non-linear registration of a given data 


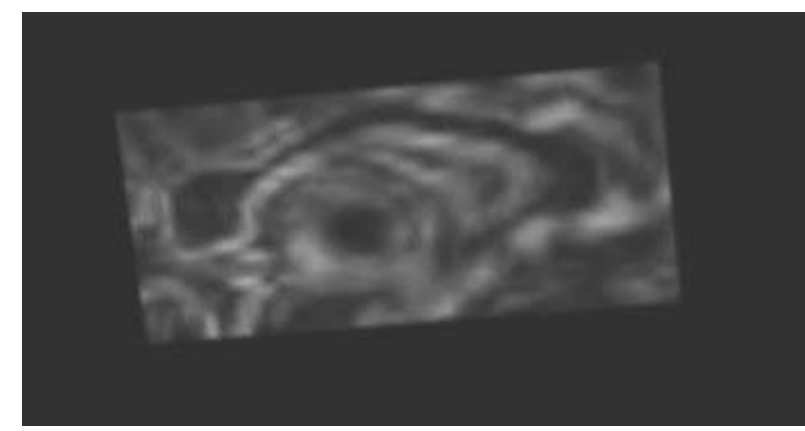

(a) Tensorial morphological gradient (TMG)

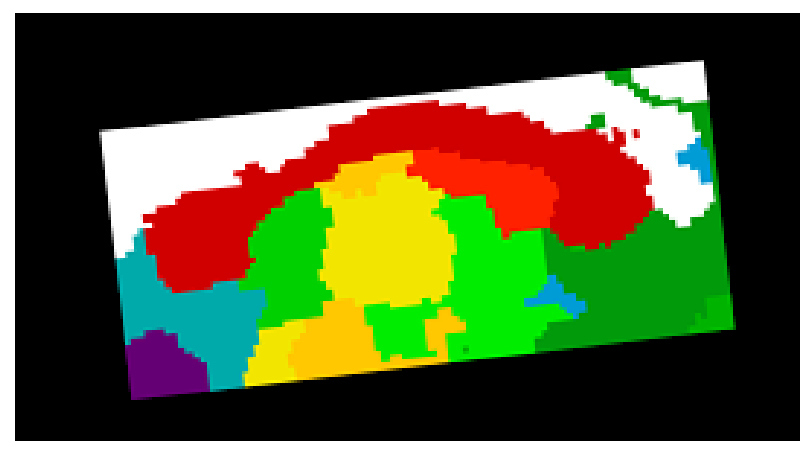

(b) Resulting labeled regions from Watershed on TMG

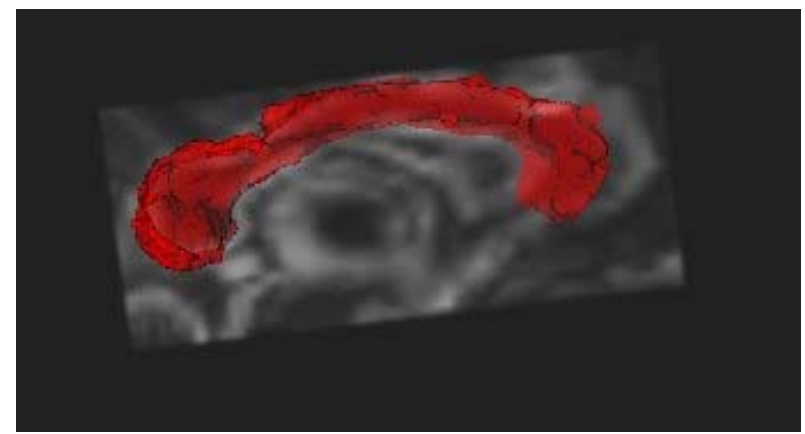

(c) Corpus callosum (3D) segmented by the watershed on the TMG

Figure 2. Segmenting the corpus callosum: the computed TMG (one slice), the hierarchical watershed transform (one slice) and the 3D segmentation result

set with a probabilistic anatomical atlas (ANIMAL). The ANIMAL (Automated Non-Linear Registration Package for Stereotaxic Transformation of MRI data) is a completely automatic method, based on multi-scale, three dimensional (3D) cross-correlation, to non-linearly register two MRI volumes together [6]. Instead of using diffusion images to perform the segmentation, it combines information from $T 1$, $T 2$ and $P D$ images to accomplish the task.

In Fig. 3 results from the TMG segmentation and from the ANIMAL+INSECT segmentation are presented. Segmentation results are similar, although the first was based on the diffusion data Fig. 3(a) and the second on the T1, T2 and $P D$ images Fig. 3(b). By plotting both segmentation results in the same figure (Fig. 3(c)) it is possible to confirm their consistency. It is important to point out that the segmentation result of the ANIMAL+INSECT method is not the ground truth, and it was used only for comparison purposes.

Fig. 4 depicts what happens when the user chooses a higher number of regions $(n=100)$. Even though the corpus callosum was segmented in three distinct regions, is still possible to distinguish it as one structure by selecting all three regions and throwing away the rest.

The same experiment was repeated with different subjects to segment the corpus callosum. In all cases, the corpus callosum was successfully segmented from DTI and the results were comparable to the results of ANIMAL+INSECT algorithm. Similar experiments were also conducted in order to segment distinct brain structures.

Fig. 5 shows the 3D segmentation result of the ventricles. Again, the TMG was computed using the Frobenius Norm as the similarity metric and a 6-connected structuring element. By choosing the same number of regions $(n=60)$ used for the corpus callosum, the proposed algorithm was able to segment the left and right ventricles. Although segmenting the ventricles could be eventually accomplished by a threshold applied in a fractional anisotropy (FA) map, their separation in right and left ventricles would not be straightforward.

Fig. 6 presents the result for the cortico-spinal tract segmentation. It was done by TMG computation using the Frobenius Norm and new markers were placed in the 120 most significant regions of the image. Again, the hierarchical watershed transform was used to perform segmentation and the obtained result is as expected.

Because there is no gold standard available for comparison, a quantitative assessment is difficult. All segmentation results were visually compared with neuroanatomical atlas, results from the ANIMAL+INSECT algorithm and results from papers cited in Subsection II-B. From this comparison it is possible to confirm that the proposed algorithm succeed segmenting the above mentioned structures.

\section{CONCLUSIONS}

This paper proposes the segmentation of brain structures by running the watershed transform over the tensorial morphological gradient (TMG) computed from diffusion tensor images. The TMG processes the tensorial information and transforms it into an scalar image, making possible the use of the hierarchical watershed to segment it. As opposed to other DTI based segmentation algorithms, the proposed method requires no manual seed placement and/or initial surface delineation. The only parameter is the number of regions in which the image should be segmented, in contrast to other methods, that require sometimes 4 parameters to perform segmentation. Experiments with real DTI were conducted, where different brain structures, such as the corpus callosum, the cortico-spinal tracts and the ventricles, were successfully segmented. 


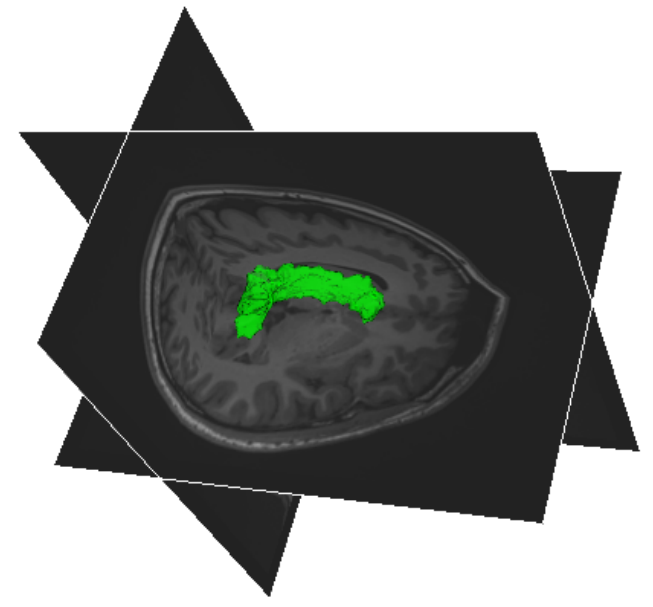

(a) $\mathrm{TMG}+$ Watershed

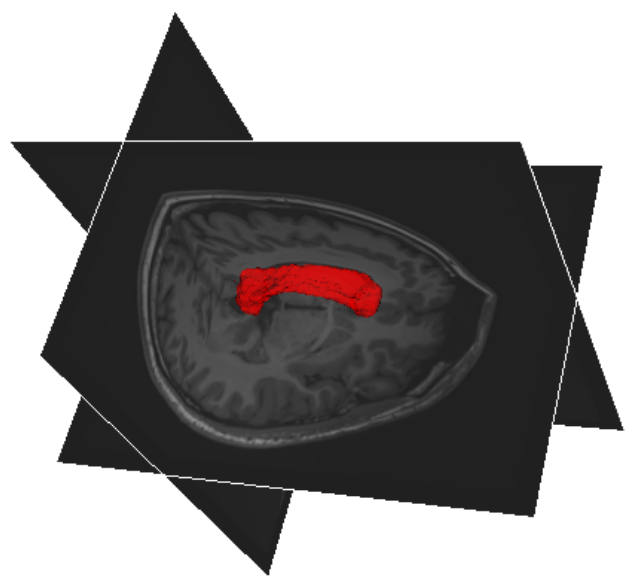

(b) ANIMAL + INSECT

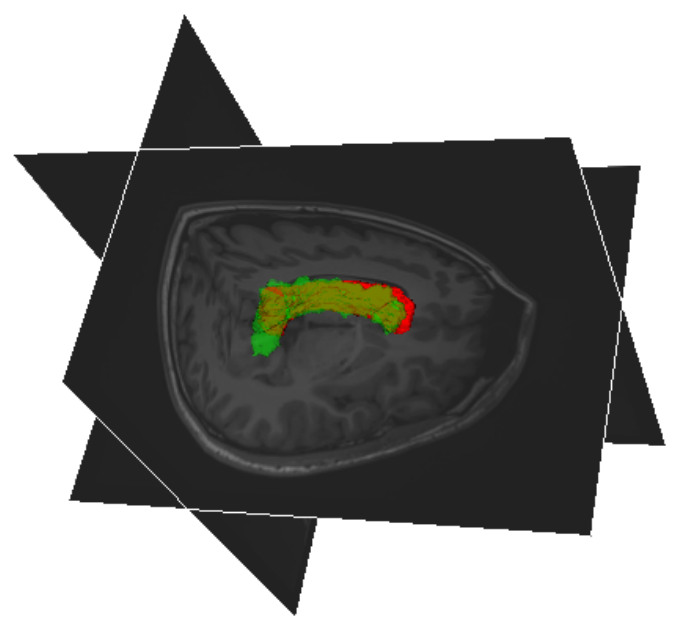

(c) Both results (overlaid)

Figure 3. Comparison of corpus callosum obtained by different segmentation methods: In green, the TMG+Watershed performed on DTI and in red, the ANIMAL+INSECT performed on T1, T2 and PD images

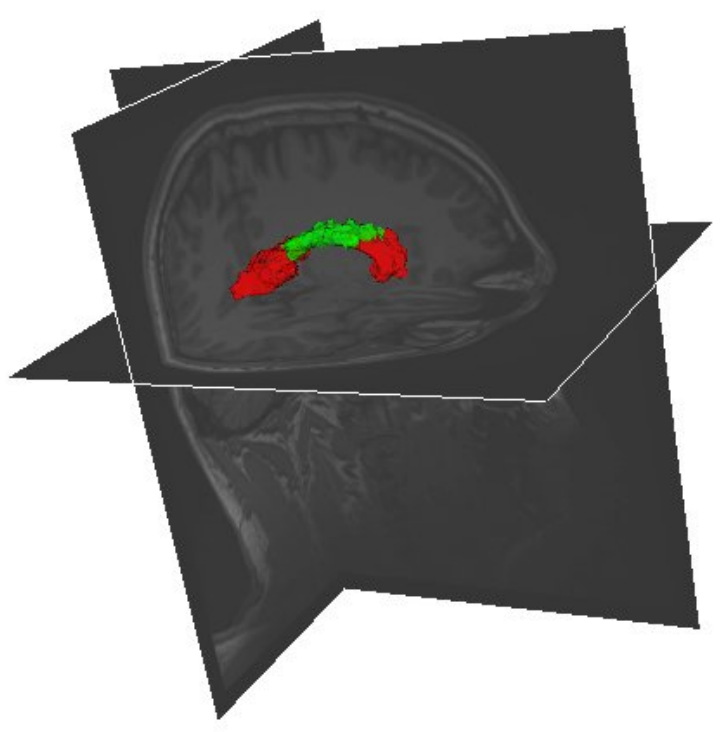

Figure 4. 3D segmentation result of corpus callosum, when increasing the number $n$ of regions. Instead of only one label, three labels were assigned to the corpus callosum by the watershed transform.

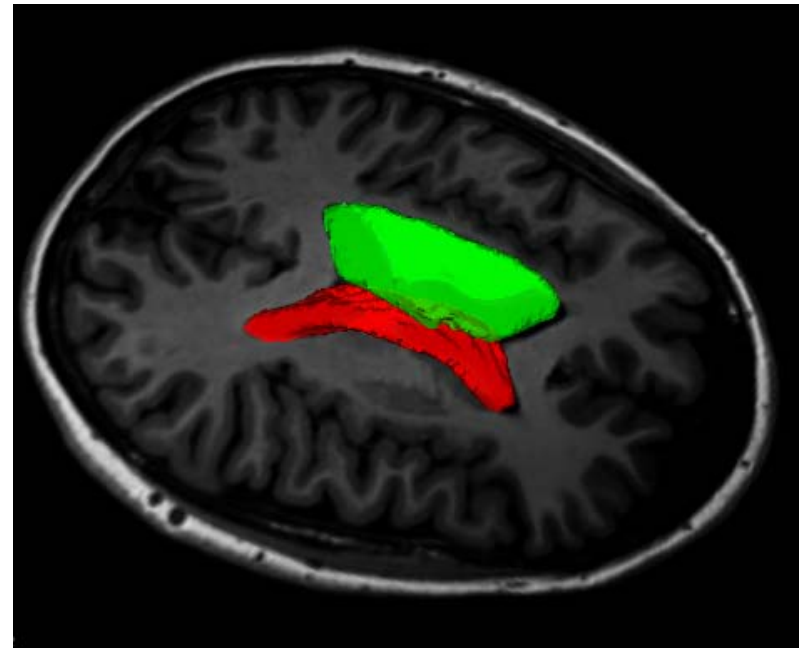

(a) ANIMAL

Figure 5. Left and right ventricles, segmented by the watershed transform on the TMG

\section{REFERENCES}

[1] C. Broit, "Optimal registration of deformed images," Ph.D. dissertation, Computer and Information Science Dept., University of Pennsylvania, Philadelphia, PA, 1981.

[2] I. Kapouleas, "Segmentation and feature extraction for magnetic resonance brain image analysis," in Proceedings of the 10th International Conference on Pattern Recognition, vol. i, 1990, pp. 583-590 vol.1.

[3] G. Gerig, J. Martin, R. Kikinis, O. Kübler, M. E. Shenton, and F. A. Jolesz, "Automating segmentation of dual-echo MR 


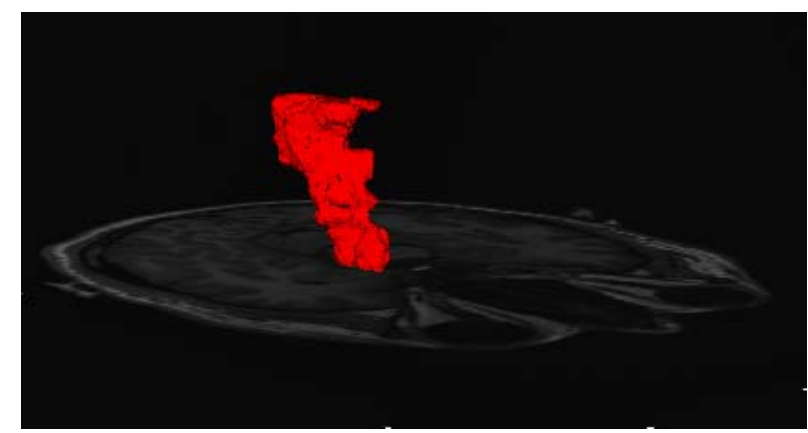

Figure 6. Segmentation of cortico-spinal tract by the proposed method

head data," in IPMI 91: Proceedings of the 12th International Conference on Information Processing in Medical Imaging. London, UK: Springer-Verlag, 1991, pp. 175-187.

[4] M. E. Brummer, R. M. Mersereau, R. L. Eisner, and R. R. Lewine, "Automatic detection of brain contours in MRI data sets," IEEE Transactions on Medical Imaging, vol. 12, no. 2, pp. 153-166, 1993.

[5] M. Miller, G. Christensen, Y. Amit, and U. Grenander, "Mathematical textbook of deformable neuroanatomies," Proceedings of the National Academy of Sciences of the United States of America, vol. 90, no. 24, pp. 11944-11 948, 1993.

[6] L. D. Collins, C. J. Holmes, T. M. Peters, and A. C. Evans, "Automatic 3-D model-based neuroanatomical segmentation," Human Brain Mapping, vol. 3, no. 3, pp. 190-208, 1995.

[7] D. L. Collins, A. P. Zijdenbos, W. F. C. Baaré, and A. C. Evans, "ANIMAL+INSECT: Improved cortical structure segmentation," in IPMI 99: Proceedings of the 16th International Conference on Information Processing in Medical Imaging. London, UK: Springer-Verlag, 1999, pp. 210-223.

[8] L. Zhukov, K. Museth, D. Breen, R. Whitaker, and A. Barr, "Level set modeling and segmentation of DT-MRI brain data," Journal of Electronic Imaging, vol. 12, pp. 125-133, 2003.

[9] Z. Wang and B. Vemuri, "DTI segmentation using an information theoretic tensor dissimilarity measure," IEEE Transactions on Medical Imaging, 2005.

[10] L. Jonasson, X. Bresson, P. Hagmann, O. Cuisenaire, R. Meuli, and J. Thiran, "White matter fiber tract segmentation in DT-MRI using geometric flows," Medical Image Analysis, vol. 9, no. 3, pp. 223-236, 2005.

[11] Y. Weldeselassie and G. Hamarneh, "DT-MRI segmentation using graph cuts," in Medical Imaging 2007: Image Processing, Proceedings of the SPIE. SPIE, 2007.

[12] Y. Y. Boykov and M.-P. Jolly, "Interactive graph cuts for optimal boundary and region segmentation of objects in ND images," International Conference in Computer Vision, vol. 01, p. 105, 2001.

[13] C. Lenglet, M. Rousson, and R. Deriche, "A statistical framework for DTI segmentation." in Proceedings of the International Symposium on Biomedical Imaging. IEEE, 2006, pp. 794-797.
[14] S. N. Niogi, P. Mukherjee, and B. D. McCandliss, "Diffusion tensor imaging segmentation of white matter structures using a reproducible objective quantification scheme (ROQS)," NeuroImage, vol. 35, pp. 166-174, 2007.

[15] L. Rittner, F. Flores, and R. Lotufo, "New tensorial representation of color images: Tensorial morphological gradient applied to color image segmentation," in SIBGRAPI07: Proceedings of the XX Brazilian Symposium on Computer Graphics and Image Processing. Belo Horizonte, Brazil: IEEE Computer Society, 2007, pp. 45-52.

[16] L. Rittner and R. Lotufo, "Diffusion tensor imaging segmentation by watershed transform on tensorial morphological gradient," in SIBGRAPI08: Proceedings of the XXI Brazilian Symposium on Computer Graphics and Image Processing. Campo Grande, Brazil: IEEE Computer Society, 2008, pp. 196-203.

[17] H. J. A. M. Heijmans, Morphological Image Operators. Boston: Academic Press, 1994.

[18] C. Pierpaoli and P. J. Basser, "Toward a quantitative assessment of diffusion anisotropy," Magnetic Resonance in Medicine, vol. 36, no. 6, pp. 893-906, 1996.

[19] D. Alexander, J. Gee, and R. Bajcsy, "Similarity measures for matching diffusion tensor images," in Proceedings of the British Machine Vision Conference, vol. 1, Nottingham, UK, pp. 93-102.

[20] A. S. D.K. Jones, M.A. Horsfield, "Optimal strategies for measuring diffusion in anisotropic systems by magnetic resonance imaging," Magnetic Resonance in Medicine, vol. 42, no. 3, pp. 515-525, 1999.

[21] M. Wiegell, D. Tuch, H. Larson, and V. Wedeen, "Automatic segmentation of thalamic nuclei from diffusion tensor magnetic resonance imaging," NeuroImage, vol. 19, pp. 391-402, 2003.

[22] U. Ziyan, D. Tuch, and C. Westin, "Segmentation of thalamic nuclei from DTI using spectral clustering," in MICCAIO6: Proceedings of the Medical Image Computing and Computer Assisted Intervention, ser. Lecture Notes in Computer Science, Denmark, 2006, pp. 807-814.

[23] S. Beucher and F. Meyer, "The morphological approach to segmentation: The watershed transformation," in Mathematical Morphology in Image Processing. Marcel Dekker, 1992, ch. 12 , pp. $433-481$.

[24] L. Vincent and P. Soille, "Watersheds in Digital Spaces: An Efficient Algorithm Based on Immersion Simulations," IEEE Transactions on Pattern Analysis and Machine Intelligence, vol. 13, no. 6, pp. 583-598, June 1991.

[25] F. Meyer, "An overview of morphological segmentation," International Journal of Pattern Recognition and Artificial Intelligence, vol. 15, no. 7, pp. 1089-1118, 2001.

[26] E. R. Dougherty and R. A. Lotufo, Hands-on Morphological Image Processing. SPIE, 2003, vol. TT59. 